\title{
Effect of electron thermal carriers on thermal diffusivity of metals
}

\begin{abstract}
A photoflash method of thermal diffusivity measurement was used to determine the value of thermal diffusivity of a sandwiched aluminum layer in a three-layer sample when the sample is simultaneously subjected to both thermal and electric field gradients. The value of the thermal diffusivity of the sample initially measured before electric field was applied agreed well with other reported results. The thermal diffusivity was also found to decrease or increase under the influence of the electric field strength or direction.
\end{abstract}

Keyword: Electron and phonon thermal carriers; Photoflash technique; Thermal diffusivity 